\title{
Homozygosity at variant $M L H 1$ can lead to secondary mutation in NF1, neurofibromatosis type I and early onset leukemia
}

\author{
Hani Alotaibi, Marie D. Ricciardone ${ }^{1}$, Mehmet Ozturk* \\ Bilkent University, Department of Molecular Biology and Genetics, 06800 Ankara, Turkey \\ Received 25 June 2007; received in revised form 30 July 2007; accepted 1 August 2007 \\ Available online 9 August 2007
}

\begin{abstract}
Heterozygous germ-line variants of DNA mismatch repair (MMR) genes predispose individuals to hereditary non-polyposis colorectal cancer. Several independent reports have shown that individuals constitutionally homozygous for $M M R$ allelic variants develop early onset hematological malignancies often associated to features of neurofibromatosis type 1 (NF1) syndrome. The genetic mechanism of NF1 associated to MMR gene deficiency is not fully known. We report here that a child with this form of NF1 displays a heterozygous $N F 1$ gene mutation (c.3721C > T), in addition to a homozygous MLH1 gene mutation (c.676C $>$ T) leading to a truncated MLH1 protein (p.R226X). The parents did not display NF1 features nor the NF1 mutation. This new NF1 gene mutation is recurrent and predicts a truncated neurofibromin (p.R1241X) lacking its GTPase activating function, as well as all C-terminally located functional domains. Our findings suggest that NF1 disease observed in individuals homozygous for deleterious MMR variants may be due to a concomitant NF1 gene mutation. The presence of both homozygous MLH1 and heterozygous NF1 mutation in the child studied here also provides a mechanistic explanation for early onset malignancies that are observed in affected individuals. It also provides a model for cooperation between genetic alterations in human carcinogenesis.
\end{abstract}

(C) 2007 Elsevier B.V. All rights reserved.

Keywords: Neurofibromatosis type 1; $M L H 1 ; N F 1$; Cooperative effect; Downstream mutation

\section{Introduction}

The classical neurofibromatosis type 1 (NF1) syndrome, also known as von-Recklinghausen's disease or peripheral neurofibromatosis, is an autosomal dominant disorder linked to NF1 gene mutations of which $50 \%$ are new mutations [1]. In 1999, Ricciardone et al. [2] and Wang et al. [3] have identified a novel form of NF1 syndrome that is associated with homozygous germ-line

\footnotetext{
* Corresponding author. Tel.: +90 312 2665081; fax: +90 3122665097 .

E-mail address: ozturk@ fen.bilkent.edu.tr (M. Ozturk).

${ }^{1}$ Present address: Foreign Affairs Officer, U.S. Department of State, Washington DC, USA.
}

mutations of $M L H 1$ gene in two independent families. These initial observations have now been confirmed by others and extended to biallelic mutations of other DNA mismatch repair (MMR) genes, including MSH2, MSH6 and PMS2 [4-8]. Eight children (five girls, three boys) homozygous for MMR gene variants have been independently described [reviewed in ref. 7]. All of these children present with clinical features of NF1 that are often associated with hematological malignancies. Four of the families have been initially identified as hereditary non-polyposis colorectal cancer (HNPCC) families $[2-4,6]$. The fifth case did not have a familial history of cancer [5]. $M L H 1$ and $M S H 2$ genes were mutated in four and one families, respectively. Homozygous mutations of $M L H 1$ gene were demonstrated in five, and suspected 
in two children. The remaining child had a homozygous MSH2 gene mutation. The novel form of NF1 is associated with early onset childhood malignancies, most of which are either leukemias or lymphomas [2,3,5]. All the parents of the affected children were heterozygous for the same MMR gene mutation, some of whom had developed colorectal cancers at the time of diagnosis, but none displayed NF1 disease and/or childhood malignancies.

NF1 with early onset childhood malignancies observed in these children is specifically associated with a homozygous MMR gene mutation. However, this association does not necessarily mean that homozygous MMR gene mutation is directly implicated in the development of this disease syndrome. NF1 which is not a known component of HNPCC syndrome [8] is not associated with MMR gene mutation [3]. However, it remains possible that a concomitant mutation affecting $N F 1$ gene is involved in this disease.

In this report, we further studied the archival genomic DNAs from a Turkish family that we have already reported for the status of $M L H 1$ [2]. In this HNPCC family, displaying a germ-line mutation in MLH1 (p.R226X), there was a consanguineous marriage between two members, both heterozygous for the mutation (Fig. 1). All three children born from this marriage developed NF1 disease associated with early onset hematological malignancies and a homozygous $\mathrm{MLHI}$ mutation had been found in at least two siblings. The mother and father were heterozygous for MLHI muta-

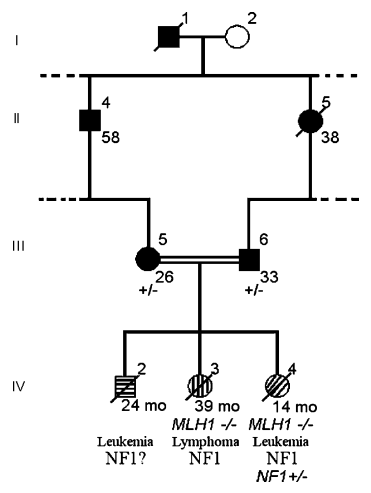

Fig. 1. Simplified pedigree of the HNPCC kindred TF3 with $M L H 1$ gene mutation. Filled symbols indicate colon cancer; shaded symbols indicate neurofibromatosis type 1 . Numbers below symbols indicate age at diagnosis. $( \pm)$ : Heterozygous $M L H 1$ mutation. The TF3 kindred was described previously [4]. Briefly, the focus of this investigation, child IV-4, developed atypical chronic myeloid leukemia at 12 months and displayed clinical signs of type I neurofibromatosis. Two siblings, IV-3 and IV-2, also developed leukemia/lymphoma and died in early childhood. Clinical signs of neurofibromatosis were confirmed in the sibling IV-3. The parents (III-5 and III-6) were both afflicted with colorectal cancer at an early age, 26 and 33 years old, respectively. tion and developed colorectal cancers at 26 and 33 years, respectively, but they did not display NF1 clinical features nor did they develop hematological malignancy [2]. We report here on the identification of a heterozygous NF1 gene mutation in the archival DNA samples from child IV-4 who developed atypical chronic myeloid leukemia at 12 months, and displayed NF1 features with café au lait spots and fibromatous skin tumors [2].

\section{Materials and methods}

\subsection{Patients and DNA samples}

Informed consent was obtained from all participants in the study. The TF3 kindred was described previously [2]. Briefly, the focus of this investigation, child IV-4, developed atypical chronic myeloid leukemia at 12 months and displayed NF1 features with more than 10 abdominal café au lait spots ( $\geq 15 \mathrm{~mm}$ ) and two fibromatous skin tumors. Two siblings, IV-3 and IV-2, also developed leukemia/lymphoma and died in early childhood. Clinical signs of neurofibromatosis were confirmed in the sibling IV-3. The parents (III-5 and III-6) were both afflicted with colorectal cancer at an early age, 26 and 33 years old, respectively. Blood samples from the parents were collected in tubes containing EDTA. The DNAs of wt, FIII-6, MIII-5 and child IV-4 has been extracted from freshly collected blood samples and was then stored at $-80^{\circ} \mathrm{C}$. The DNA for sibling IV-3 has been extracted from an archival bone marrow smear. Differential blood counting of the child IV-4 at the time the blood was drawn for DNA analysis displayed $14 \%$ blast cells [2].

\subsection{PCR and SSCP}

NF1 exons 16, 21, 22, 23-2 and 23a were amplified using specific primers that were described previously [9]. PCR was carried out in $25 \mu \mathrm{l}$ reaction volumes containing $1.5 \mathrm{mM}$ $\mathrm{MgCl}_{2}, 10$ pmoles of each primer, $200 \mu \mathrm{M}$ dNTP mix and 1 unit Taq DNA polymerase (Fermentas) and 50-100 ng genomic DNA. PCR conditions were an initial denaturation step at $95^{\circ} \mathrm{C}$ for $5 \mathrm{~min}$ followed by 30 cycles of $30 \mathrm{~s}$ at $95^{\circ} \mathrm{C}, 30 \mathrm{~s}$ at $55^{\circ} \mathrm{C}$, $30 \mathrm{~s}$ at $72^{\circ} \mathrm{C}$ followed by a $10 \mathrm{~min}$ final extension step. Quality of PCR products was verified by agarose gel electrophoresis. For single strand conformation polymorphism (SSCP) analysis, PCR was done in the presence of $1 \mu \mathrm{Ci}\left[{ }^{32} \mathrm{P}\right] \mathrm{dCTP}$ (Amersham) per reaction and radiolabeled $\mathrm{PCR}$ products were denatured using SSCP gel loading buffer (95\% formamide (Sigma), $10 \mathrm{mM} \mathrm{NaOH}$ (Carlo Erba), $0.25 \%$ bromophenol blue (Sigma) and $0.25 \%$ xylene cyanol (Sigma)). The DNA solution was heated at $95^{\circ} \mathrm{C}$ for $2 \mathrm{~min}$ and kept on ice for at least $10 \mathrm{~min}$ before loading onto a $6 \%$ [75:1] acrylamide gel on the EC 160 apparatus. Electrophoresis was performed at $45 \mathrm{~W}$ constant power at $4{ }^{\circ} \mathrm{C}$ for $8 \mathrm{~h}$. Phosphoimaging was performed using the Molecular Imager ${ }^{\circledR}$ System, GS-525 (Bio-Rad). The dried gel was placed into the cassette and a clean, erased screen was then placed over the gel. After exposure, the screen was scanned and the image was captured using Multi-Analyst software. 


\subsection{DNA sequence analysis}

Sequence analysis was performed using DYEnamic ET Terminator cycle sequencing kit (Amersham) according to the recommended protocol with the following modifications: annealing temperature was $59^{\circ} \mathrm{C}$, and only 3 pmoles of primer were used for the reverse primer reaction. Automated sequence analysis was performed on the 310 Genetic Analyzer (ABI; Perkin-Elmer) as recommended by the manufacturer.

\subsection{TaqI restriction digestion}

Genomic DNAs from parents (MIII-5 and FIII-6), child IV-4 and a wild-type control were used to amplify NF1 exon 22. The amplified products were analyzed by electrophoresis, and normalized by densitometric analysis using the BioRad Multi-Analyst software. Equal amounts of PCR products were used for TaqI digestion, which was performed at $65^{\circ} \mathrm{C}$ under mineral oil. The restriction digestion products with undigested controls were resolved on a $4 \%$ NuSieve $3: 1$ agarose (FMC Bioproducts) prepared in $0.5 \times \mathrm{TBE}$, for $3 \mathrm{~h}$ at $8 \mathrm{~V} / \mathrm{cm}$. Densitometric quantification was performed on the undigested DNA fragments, the amount of the mutant allele resistant to restriction enzyme digestion was compared to the undigested PCR product.

\section{Results and discussion}

Archival genomic DNA from child IV-4 was subjected to a detailed $N F 1$ gene mutation analysis (Fig. 1). As $N F 1$ is a large gene, we applied a rapid screening technique based on SSCP analysis of selected NF1 gene regions. The exons that contained microsatellites and $\mathrm{CpG}$ islands within the NF1 GAP-related domain (NF1-GRD) were targeted initially (Fig. 2).

Following the screening of exons 16, 21, 22, 232 and 23a, we obtained a band-shift with exon 22 in DNA from child IV-4 that was not present in parental DNAs. PCR products corresponding to other exons did not produce band-shifts (data not shown). An independently prepared PCR product for exon 22 was then used for direct DNA sequence analysis. We identified a $\mathrm{C}$

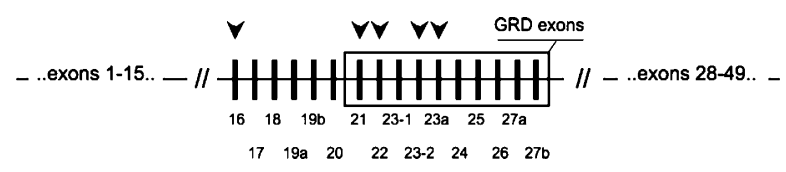

Fig. 2. Schematic representation of $N F 1$ exons used in mutation screening experiments. NF1 exons selected for analysis were amplified from genomic DNA, and PCR products were further analyzed by SSCP and the presence of the mutation was then confirmed by direct DNA sequence analysis. Vertical lines represent $N F 1$ exons, transparent rectangle represents NF1-GRD exons, and arrowheads mark exons selected for analysis. Drawing is not to scale. to $\mathrm{T}$ transition at nucleotide 3721 of $N F 1$ gene. The electropherogram profile obtained displayed both wildtype and mutant sequences, suggesting that child IV-4 had a unique heterozygous NF1 mutation. Both father and mother displayed only wild-type NF1 sequence at this site, indicating that child IV-4 had a new NFI gene mutation (Fig. 3a). This mutation is predicted to cause a substitution of arginine 1241 for a stop codon; (p.R1241X) at the protein level. The point mutation was verified by restriction enzyme digestion with TaqI, whose restriction site is lost as a consequence of the $\mathrm{C}$ to $\mathrm{T}$ transition. Restriction enzyme digestion experiments indicated that only $50 \%$ of the PCR product from child IV-4 was digested with TaqI, clearly confirming the presence of a heterozygous state in this exon. In contrast, PCR products from father, mother as well as from a control sample were completely digested by TaqI, as expected from the DNA sequence data (Fig. 3b).

To rule out the possibility of incomplete digestion from the mutant allele of child IV-4 sample, we amplified a longer DNA fragment using another intronic primer located $1438 \mathrm{bps}$ upstream of the priming site of exon 22, harboring an additional TaqI restriction site. The PCR products $(1770 \mathrm{bp})$ from both parents and both siblings (child IV-3 and child IV-4) were digested to completion with TaqI, as a result, the presence of the (c.3721C > T) heterozygous state was confirmed for child IV-4 by the loss of TaqI recognition site (see supplementary figure). This mutation was absent from a sister (child IV-3), further confirming that child IV-4 had a new NFI mutation.

Since the initial description of the association of neurofibromatosis features and early onset of childhood malignancies with homozygous mutations of $\mathrm{MLH}$ gene [2,3], 14 cases of biallelic mismatch repair gene mutations (mostly homozygous, but also compound heterozygous) affecting the $M L H 1, M S H 2, M S H 6$ or PMS2 genes have been described, as recently reviewed by Bandipalliam [8]. These cases are characterized by early onset hematological malignancies (mean age of diagnosis: 5.6 years), and gastrointestinal cancers (mean age of diagnosis: 12.2 years), as well as the distinct clinical features of type I neurofibromatosis [8]. More importantly, such clinical features have not been detected in the parents of these individuals [8], strongly suggesting that this particular form of neurofibromatosis is caused by mismatch repair deficiency leading to a mutation of NF1 gene. Several studies provided convincing data supporting the hypothesis that MMR deficiency and neurofibromatosis may be associated with NF1 mutations. Wang et al. [10] have reported that NF1 gene is often mutated in DNA mismatch repair-deficient cancer cell 
(a)
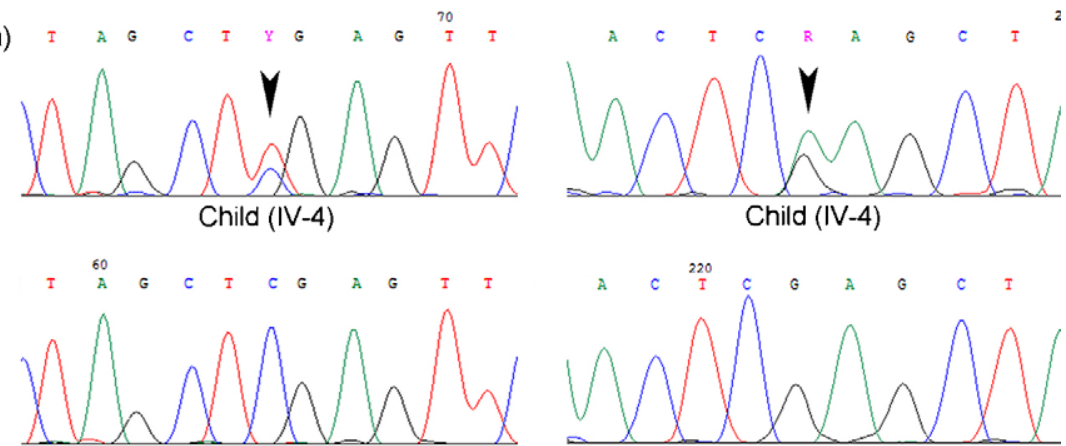

Father (III-6)

Father (III-6)

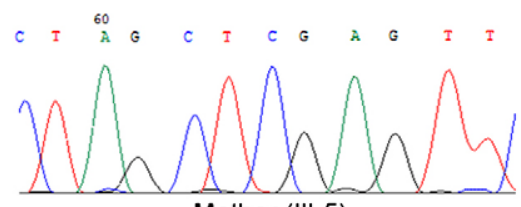

Mother (III-5)

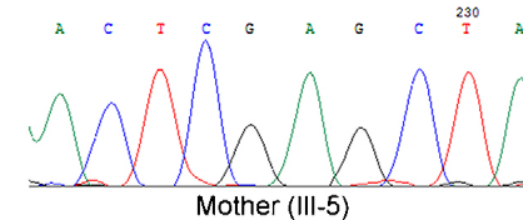

(b)

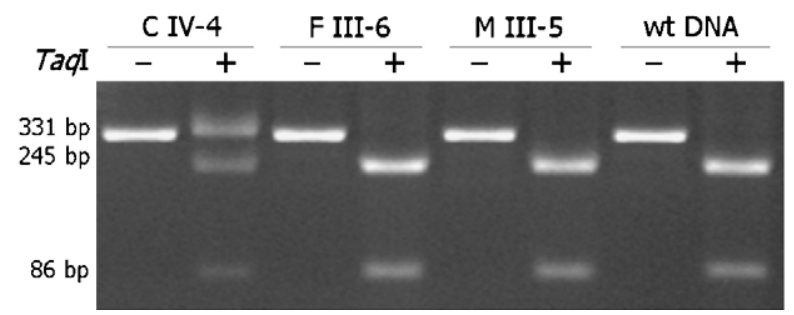

Fig. 3. Heterozygous NF1 mutation in a MLH1-deficient child with neurofibromatosis type I and early onset leukemia. Genomic DNAs from parents (MIII-5 and FIII-6), child IV-4 and a wild-type control were used to amplify NF1 exon 22 to be used for DNA sequence analysis and then for verification by restriction enzyme digestion. (a) A small stretch of the electropherogram flanking the mutation showing the nucleotide sequence obtained with forward and reverse primers (the left and right panels, respectively). Arrow heads indicate heterozygosity [T/C designated as Y] at nucleotide 3721. (b) PCR amplified DNA samples were analyzed by electrophoresis, and normalized by densitometric analysis using the BioRad multi-analyst software. Equal amounts of PCR products were used for TaqI digestion (lanes with "+" signs) as described in Section 2. The enzyme's digestion products of wild-type sequence are 245 and $86 \mathrm{bp}$, while the c.3721C > T mutation will render the site unrecognizable by the enzyme. Lane C IV-4, child IV-4; F III-6, Father; M III-5, Mother; wt DNA, wild-type control. Data represents experiments repeated at least three times with freshly prepared PCR products.

lines, as well as in primary tumors exhibiting microsatellite instability. They have also shown a "mosaic" type of mutation of the murine $n f 1$ gene in 2 of 14 clones generated from $m l h l$-deficient mouse embryonic fibroblasts, providing additional evidence that $N F 1$ gene may be a mutational target in mismatch repair-deficient cells [10]. Moreover, leukemogenesis in $n f 1$ heterozygous mice was shown to be accelerated by mlhl deficiency [11]. However, to our knowledge, there is no previous data reporting potential $N F 1$ gene mutations in individuals with DNA mismatch repair gene mutations [7]. Thus, the molecular mechanism(s) of neurofibromatosis in individuals with biallelic mutations remained unknown. Here, we show that blood DNA from a child who developed atypical chronic myeloid leukemia at 12 months and displayed NF1 features with more than 10 abdominal café au lait spots $(>15 \mathrm{~mm})$ and two fibromatous skin tumors displays a new c. $3721 \mathrm{C}>\mathrm{T}$ mutation at $N F 1$ gene. This is a recurrent mutation at a predicted $\mathrm{CpG}$ site, initially reported by Fahsold et al. [12], leading to a severely truncated neurofibromin protein (p.R1241X). The mutant protein is expected to have lost its negative regulatory function of ras oncogene because of the lack of GTPase activating as well as other C-terminally located functional domains [13]. To our knowledge this is the first demonstration of a NFI gene mutation in a MMR deficient patient with neurofibromatosis and early onset hematological malignancy. Our finding provides a plausible explanation for the genetic cause of neurofibromatosis observed in patients displaying this phenotype [8]. Thus, secondary NF1 mutations may predispose MMR deficient individuals to neurofibromatosis and/or early onset malignancies, similar to classical NF1 syndrome [12]. 
Currently, the genetic mechanism of $N F 1$ mutation in MMR deficient individuals is unknown. NF1 is known as an autosomally dominant condition. About $50 \%$ of cases result from new mutations, and some cases display germline mosaicism. Most of the new mutations occur on the paternally derived chromosome, whereas large deletions are usually of maternal origin [14]. The $\mathrm{C}$ to $\mathrm{T}$ mutation observed in the child IV-4 occurred at a $\mathrm{CpG}$ motif, such type of mutations are known to occur as a result of deamination of methyl cytosine which would lead to a T:G mismatch which is usually repaired by MMR system. As this mutation occurred in a MMR deficient condition, it may be secondary to this particular condition. It is unclear whether this mutation occurred post-zygotically in the parental chromosome, or rather somatically in the affected child. Such mutations appear to occur in NF1 patients as some of them display a "mosaic" pattern $[12,14]$. If the same event took place in the child IV-4, this must have been a very early embryonic mutation, as the child already displayed many café-au-lait spots $(\geq 15 \mathrm{~mm})$ and two fibromatous skin tumors at the age of 12 months, together with leukemia [2]. Although we were unable to test whether this particular mutation display "mosaicism", we favor the hypothesis that the heterozygous NF1 mutation detected in child IV-4 is a very early somatic mutation that occurred in the embryonic cells.

The co-existence of a homozygous MMR gene mutation with a tumor suppressor gene mutation (i.e. NFI mutation) at the individual level is also a new finding. This has been previously suggested as a potential mechanism involved in cancer predisposition of individuals carrying constitutional MMR gene mutations $[2,3]$. Accordingly, the loss of the wild-type MMR gene in pre-malignant cells results in genetic instability giving rise to a high rate of mutations eventually affecting critical tumor suppressor genes [15]. As already indicated, the NF1 gene appears as a frequent target for mutations in MMR-deficient cells [10]. Therefore, the occurrence of the new NF1 mutation identified here may have been facilitated by DNA mismatch repair deficiency in body cells due to inherited homozygous $M L H 1$ mutation in the affected child. However, this remains as an interesting, yet unproven hypothesis in the absence of a direct evidence for a cause-effect relationship for the co-occurrence of $M L H 1$ and NF1 gene mutations in the same individual.

Cancer is a multistage process which requires the contribution of different genetic alterations. Mouse models have been successfully used to study carcinogenic effects resulting from combinations of genetic alterations. Such studies have allowed developing several important concepts for tumorigenesis, such as cooperation between oncogenes, or oncogene-tumor suppressor gene interactions [16]. However, it is unknown how and to what extent such fundamental concepts apply to tumorigenesis processes in humans. Our observations provide a unique opportunity to address this issue. In the child we studied here, there was a homozygous $M L H 1$ mutation and a heterozygous NF1 mutation. A similar situation has been created experimentally in mice homozygous for $m l h 1$ (-l-) and heterozygous $n f l( \pm)$ mutations [11]. These double mutant mice displayed accelerated myeloid leukemogenesis when compared to mice carrying single gene mutations, indicating that two genetic alterations cooperated for the generation of hematological malignancies. In humans with classical NF1 syndrome due to NF1 mutations, hematological malignancies are detected, but only rarely (less than 5\%; [17]), suggesting that NF1 mutation alone has a moderate effect on hematological malignancy susceptibility. On the other hand, hematological malignancies are not a known component of tumors observed in HNPCC patients with heterozygous DNA mismatch repair gene mutations, including the parents of the child IV-4. In contrast, the child IV-4 with MLHI (-/-)/NFI ( \pm ) developed leukemia at the age of 1 year. Thus, in line with the predictions based on data gathered using experimental mice models, multiple genes may also cooperate in humans for accelerated tumor susceptibility. Child IV4 is not an isolated case; at least a dozen other children share exactly the same clinical features of early onset hematological malignancies and neurofibromatosis [8]. It will be interesting to know whether our gene alteration cooperation model is a general feature of this particular syndrome.

\section{Acknowledgements}

We thank the TF3 family members for their cooperation. We also thank Yasemin Doruk and Bilge Özbayoğlu for technical assistance.

\section{Appendix A. Supplementary data}

Supplementary data associated with this article can be found, in the online version, at doi:10.1016/j.mrfmmm. 2007.08.003.

\section{References}

[1] S.M. Huson, D.A. Compston, P. Clark, P.S. Harper, A genetic study of von Recklinghausen neurofibromatosis in south east 
Wales. I. Prevalence, fitness, mutation rate, and effect of parental transmission on severity, J. Med. Genet. 26 (1989) 704-711.

[2] M.D. Ricciardone, T. Ozcelik, B. Cevher, H. Ozdag, M. Tuncer, A. Gurgey, O. Uzunalimoglu, H. Cetinkaya, A. Tanyeli, E. Erken, M. Ozturk, Human MLH1 deficiency predisposes to hematological malignancy and neurofibromatosis type 1, Cancer Res. 59 (1999) 290-293.

[3] Q. Wang, C. Lasset, F. Desseigne, D. Frappaz, C. Bergeron, C. Navarro, E. Ruano, A. Puisieux, Neurofibromatosis and early onset of cancers in hMLH1-deficient children, Cancer Res. 59 (1999) 294-297.

[4] S. Vilkki, J.L. Tsao, A. Loukola, M. Poyhonen, O. Vierimaa, R. Herva, L.A. Aaltonen, D. Shibata, Extensive somatic microsatellite mutations in normal human tissue, Cancer Res. 61 (2001) 4541-4544.

[5] D. Whiteside, R. McLeod, G. Graham, J.L. Steckley, K. Booth, M.J. Somerville, S.E. Andrew, A homozygous germ-line mutation in the human MSH2 gene predisposes to hematological malignancy and multiple cafe-au-lait spots, Cancer Res. 62 (2002) 359-362.

[6] T.E. Raevaara, A.M. Gerdes, K.E. Lonnqvist, A. TybjaergHansen, W.M. Abdel-Rahman, R. Kariola, P. Peltomaki, M. Nystrom-Lahti, HNPCC mutation MLH1 P648S makes the functional protein unstable, and homozygosity predisposes to mild neurofibromatosis type 1, Genes Chromosomes Cancer 40 (2004) 261-265.

[7] J.R. Ostergaard, L. Sunde, H. Okkels, Neurofibromatosis von Recklinghausen type I phenotype and early onset of cancers in siblings compound heterozygous for mutations in MSH6, Am. J. Med. Genet. 139 (2005) 96-105.

[8] P. Bandipalliam, Syndrome of early onset colon cancers, hematologic malignancies \& features of neurofibromatosis in HNPCC families with homozygous mismatch repair gene mutations, Familial Cancer 4 (2005) 323-333.

[9] Y. Li, P. O'Connell, H.H. Breidenbach, R. Cawthon, J. Stevens, G. Xu, S. Neil, M. Robertson, R. White, D. Viskochil, Genomic organization of the neurofibromatosis 1 gene (NF1), Genomics 25 (1995) 9-18.

[10] Q. Wang, G. Montmain, E. Ruano, M. Upadhyaya, S. Dudley, R.M. Liskay, S.N. Thibodeau, A. Puisieux, Neurofibromatosis type 1 gene as a mutational target in a mismatch repair-deficient cell type, Hum. Genet. 112 (2003) 117-123.

[11] D.H. Gutmann, E. Winkeler, O. Kabbarah, N. Hedrick, S. Dudley, P.J. Goodfellow, R.M. Liskay, Mlh1 deficiency accelerates myeloid leukemogenesis in neurofibromatosis 1 (Nf1) heterozygous mice, Oncogene 22 (2003) 4581-4585.

[12] R. Fahsold, S. Hoffmeyer, C. Mischung, C. Gille, C. Ehlers, N. Kucukceylan, M. Abdel-Nour, A. Gewies, H. Peters, D. Kaufmann, A. Buske, S. Tinschert, P. Nurnberg, Minor lesion mutational spectrum of the entire NF1 gene does not explain its high mutability but points to a functional domain upstream of the GAP-related domain, Am. J. Hum. Genet. 66 (2000) 790-818.

[13] K. Scheffzek, M.R. Ahmadian, L. Wiesmuller, W. Kabsch, P. Stege, F. Schmitz, A. Wittinghofer, Structural analysis of the GAP-related domain from neurofibromin and its implications, EMBO J. 17 (1998) 4313-4327.

[14] S.A. Rasmussen, J.M. Friedman, NF1 gene and neurofibromatosis 1, Am. J. Epidemiol. 151 (2000) 33-40.

[15] K.W. Kinzler, B. Vogelstein, Lessons from hereditary colorectal cancer, Cell 87 (1996) 159-170.

[16] T. Van Dyke, T. Jacks, Cancer modeling in the modern era: progress and challenges, Cell 108 (2002) 135-144.

[17] N. Reed, D.H. Gutmann, Tumorigenesis in neurofibromatosis: new insights and potential therapies, Trends Mol. Med. 7 (2001) $157-162$. 